\title{
BEPCII INTERACTION REGION DESIGN AND CONSTRUCTION STATUS
}

\author{
Y. Z. Wu ${ }^{1)}$, C. H. Yu, F. S. Chen, J. B. Pang, J. F. Zhang, M. T. Wang, Q. L. Peng, X. W. Dai, \\ Y. Yang, Z. S. Yin, IHEP, Beijing, China
}

\section{Abstract}

BEPC (Beijing Electron Positron Collider) is now upgrading to a double-ring collider which requires a new storage ring to be built in the existing tunnel. A new and compact interaction region (IR) has been designed to allow a peak luminosity of $10^{33} \mathrm{~cm}^{-2} \mathrm{~s}^{-1}$ from an equal beam energy of $1.89 \mathrm{GeV}$, a crossing angle, 93 bunches with maximum beam current of $0.91 \mathrm{~A}$.

The multi-purpose superconducting magnets and conventional dual aperture quadrupoles are used as final focusing quadrupole in the interaction region. The two beams collide at the interaction point with a crossing angle of $\pm 11 \mathrm{mrad}$ and further beams separation is enhanced with the help of a septum bending magnet which locates just beyond the superconducting magnet and acts on the outgoing beam lines only. This paper will describe the IR design and the construction status.

\section{INTRODUCTION}

The circumference of BEPCII storage ring is $238 \mathrm{~m}$ and the length of IR is only about $\pm 14 \mathrm{~m}$ around the interaction point (IP). Since about 20 main magnets and other equipments must be installed into the IR, the space is very tight.

The construction of the BEPCII program is now underway and will be completed in 2006. At present, all the components of the IR are under fabrication. The coils winding of the first superconducting magnet have been completed and the cold testing in the vertical dewar are underway. The prototype of Q1a, Q1b and ISPB magnets have been successful built and their field quality meet the requirements of the beam dynamics. The studies on the fabrication techniques for special vacuum chambers have been completed and fabrications are underway.

\section{INTERFACE BETWEEN DETECTOR AND ACCELERATOR}

The interface between detector (BESIII) and accelerator is shown in Fig 1.

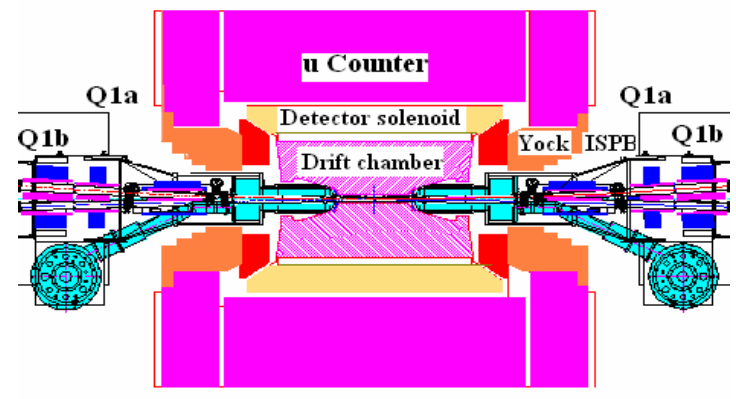

Figure 1: Interface between the BESIII and accelerator
The central part of BESIII detector is cylindrical drift chamber surrounded by an electromagnetic calorimeter, which is immersed in a superconducting solenoid field with a length of $3.6 \mathrm{~m}$ and strength of $1.0 \mathrm{~T}$.

The geometry of the drift chamber has a direct bearing on the IR design. It requires the accelerator components inside the detector must fit within a conical space with an opening angle of $21.5^{\circ}$. The first accelerator element can only approach to $0.55 \mathrm{~m}$ on each side of the IP.

\section{BEAM-LINE LAYOUT IN THE IR}

The beam line layout ${ }^{[1]}$ in the central part of the IR is shown in Fig. 2. On each side of the IP, a doublet of quadrupole is used to provide the focusing optics at the IP. The first vertical focusing quadrupole is a superconducting magnet, which is shared by both beams. Two beams collide with a horizontal crossing angle of 22 mrad at the IP. A horizontal bending magnet labelled ISPB $^{[2]}$, which is located just beyond the superconducting magnet will enhance the separation between electron beam and positron beam. ISPB is a septum magnet with a narrow septum coil. It is $2.3 \mathrm{~m}$ away from the IP with the length of $0.6 \mathrm{~m}$ and the field strength of $4 \mathrm{kGs}$. It acts on the outgoing beam-line only.

The second element of the doublet is horizontal focusing quadrupoles Q1a and Q1b.

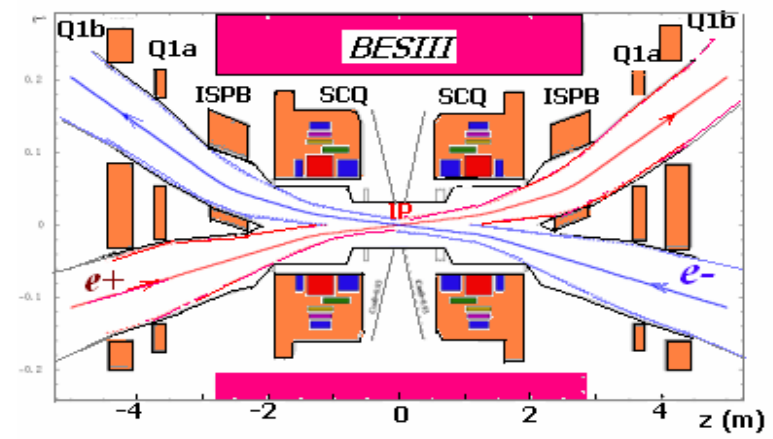

Figure 2: Layout of the interaction region

In order to achieve the aims of keeping the symmetrical structure of two rings and saving space, the quadrupoles Q1a and Q1b are designed as a two-in-one type in which the two separate beam channels for the incoming and outgoing beams have same field strength.

The separation between two beams is about $185 \mathrm{~mm}$ at the inboard face of Q1a and $231 \mathrm{~mm}$ for Q1b while the beam stay clear (BSC) is $95 \mathrm{~mm}$ and $102 \mathrm{~mm}$ respectively, so the space for the design of septum is limited. The following accelerator elements are quadrupoles Q2, Q3 and Q4 where the separation of two beams is large enough to allow them to be installed side by side into the two rings. 
With this IR layout the vertical beta function at the IP could be $1.5 \mathrm{~cm}$., as well as $1.0 \mathrm{~m}$ horizontally. The first parasitic crossing is $1.2 \mathrm{~m}$ away from the IP where the separation of two beams is about $40 \sigma$.

\section{SUPERCONDUCTING MAGNETS}

The superconducting magnet ${ }^{[3]}$ has a multi-function coil pack. As shown in Fig. 3, it consists of independent quadrupole (SCQ), horizontal dipole (SCB), vertical dipole (VDC), skew quadrupole (SKQ) and three part anti-solenoid (AS1-3) windings. The cryostat has a warm bore with an inner diameter of $\phi 132 \mathrm{~mm}$ and outer diameter of $\phi 326 \mathrm{~mm}$. The endcan of cryostat has an outer diameter of $\phi 640 \mathrm{~mm}$.

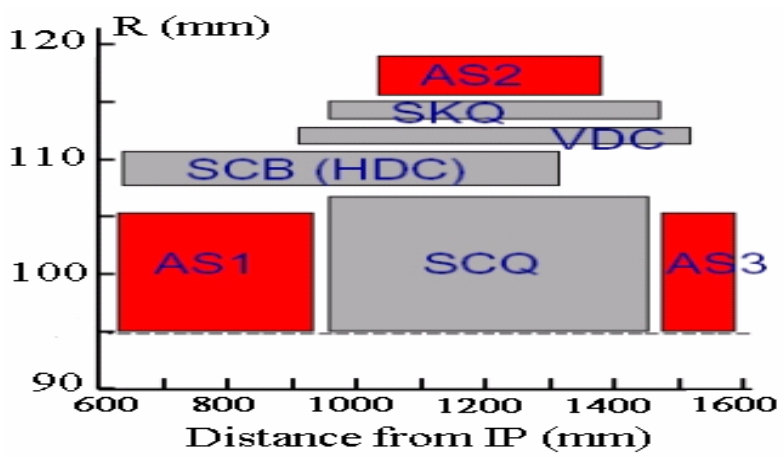

Figure 3: Coils layout of the SC magnet

The design and fabrication of the superconducting magnet are being carried out at BNL. The coils winding of the first superconducting magnet have been completed and the cold testing in a vertical dewar are underway.

\section{TWO-IN-ONE QUADRUPOLE Q1A}

The first Q1a magnet ${ }^{[4]}$ has been successfully fabricated in the factory of IHEP. Fig. 4 displays the shape of Q1a. The excitation test was succeeded up to an enough current of $1550 \mathrm{~A}$. The average temperature rise of the magnet is $20^{\circ} \mathrm{C}$ degree at the current of $1550 \mathrm{~A}$ when the flow rate of water was $5.0 \mathrm{~L} / \mathrm{min}$. The coil protection systems such as thermal relay, water flow relay, and fast voltage search device have been used on the magnet.

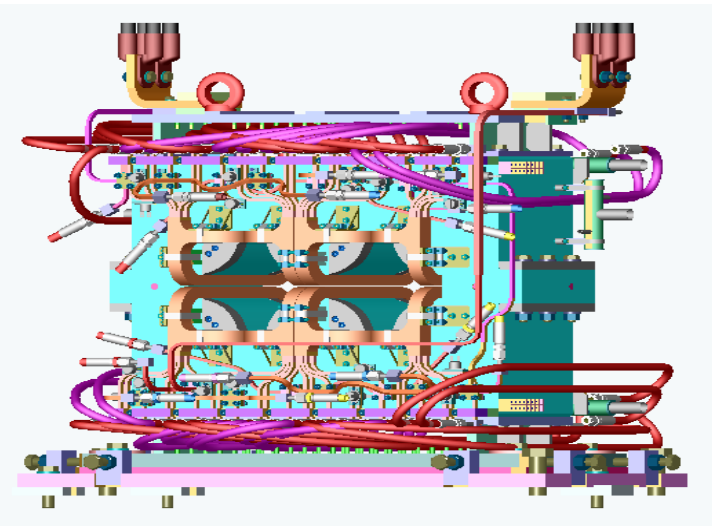

Figure 4: The shape of the Q1a magnet
The magnet is shimmed with iron sheets to achieve multipole components below $3.5 \times 10^{-4}$ at reference radius of $53.45 \mathrm{~mm}(14 \sigma)$ with a pole end chamfers $(7 \times 57)$.

\section{COMPENSATION OF THE DETECTOR SOLENOID FIELD}

The BESIII detector solenoid has the maximum field strength of $1.0 \mathrm{~T}$ over an effective length $\pm 1.8 \mathrm{~m}$ around the IP. This longitudinal field inside the beam pipe should be compensated to avoid strong coupling of the betatron oscillation.

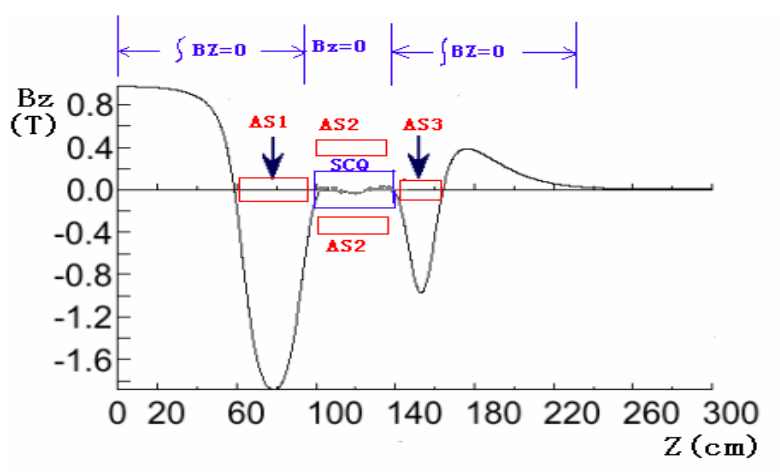

Figure 5: Distribution of the axial field $B_{z}$ of the IR

Three anti-solenoids AS1, AS2 and AS3 will be used to compensate the detector solenoid fields along the detector axis. The AS1 will balance the integral longitudinal field from the IP to the SCQ, AS2 approximately null the field over the active of SCQ and AS3 to balance the integral field in the fringe region beyond where the cold mass end. A skew quadrupole included in the SC magnet package will be used for fine tuning. Fig.5 shows the distribution of the axial field $B_{z}$ of the IR.

\section{IR VACUUM CHAMBER}

To ensure adequate quantum lifetime, the chambers are designed to accommodate at least $14 \sigma+2 \mathrm{~mm}$ in the IR region. The majority of the IR chamber ${ }^{[5]}$ is made of oxygen-free copper. The central part of the beam pipe is a double-wall beryllium structure with $0.8 \mathrm{~mm}$ gap as a cooling channel. It is $\pm 0.15 \mathrm{~m}$ long around the IP with inner diameter of $63 \mathrm{~mm}$. The inside wall of Be pipe will be coated by gold. The Be pipe is welded to a copper cylinder with the length of $0.55 \mathrm{~m}$, which is connected to SC magnet chamber through special CF63 bellows. The profile of the IP vacuum chamber is shown in Fig. 6.

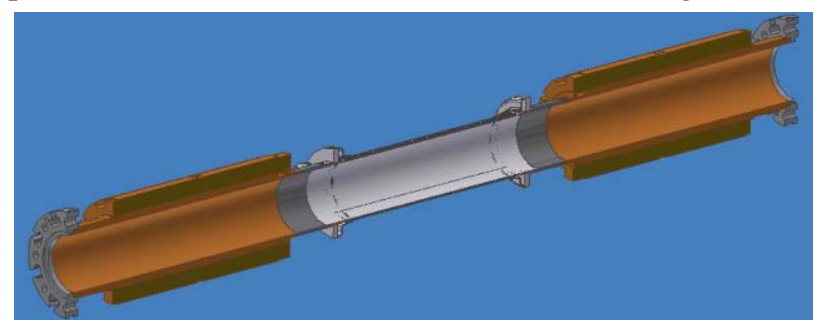

Figure 6: The profile of IP vacuum chamber. 
The SCQ chamber is about $1.24 \mathrm{~m}$ long with an inner diameter of $111 \mathrm{~mm}$. It is connected to the Y-Type chambers through special CF100 flange. An 8-button electrodes BPM is embedded before the bellows. The Ytype chamber is about $0.3 \mathrm{~m}$ long in which a bellows, one NEG pump, and two BPMs are installed. The simulations indicate that about $1.4 \mathrm{KW}$ of $\mathrm{HOM}$ power will generate in the crotch region so that water cooling channels must be installed in this section ${ }^{[6]}$. The detailed design of $\mathrm{Y}$ type vacuum chamber is shown in Fig. 7.

At the end of the Y-type section the vacuum chambers are completely separated for each beam. Both Q1a and Q1b chamber have an inner diameter of $104 \mathrm{~mm}$. After Q1b the vacuum chamber has a transition to the standard chamber.

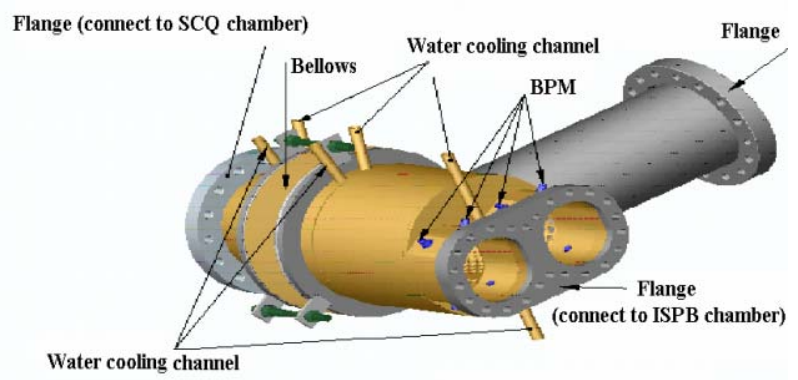

Figure 7: The detailed design of Y-type vacuum chamber

\section{SYNCHROTRON RADIATION}

Synchrotron radiation fans in the IR are mainly generated by final bending magnet OWB and SC magnet $\mathrm{SCQ}^{[1]}$. The distribution of SR fans is shown in Fig. 8. The SR generated by upstream magnets of the IP will go through the Be pipe region without hitting local surfaces. However, the two SR fans will contribute $36 \mathrm{~W}$ of SR power on the downstream surfaces of $0.5 \mathrm{~m}$ to $0.7 \mathrm{~m}$ from the IP. The other SR from these fans travel out of the near IR and is absorbed on the surfaces at $3 \sim 4 \mathrm{~m}$ from the IP.

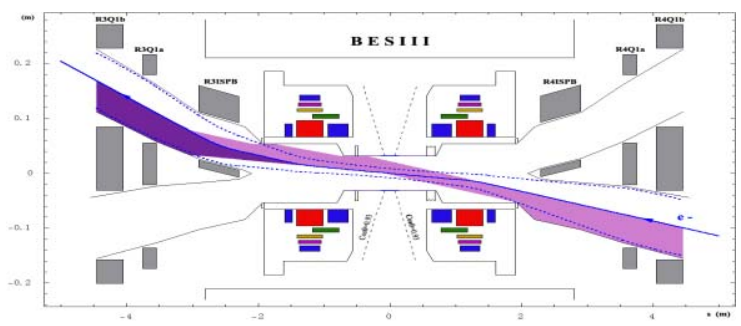

Figure 8: The distribution of SR fans

The synchrotron radiation power $(490 \mathrm{~W})$ generated by downstream magnet SCQ and ISPB hits the beam pipe at $3 \sim 4.4 \mathrm{~m}$ from the IP. A pair of masks are designed and placed in the front of the ISPB magnet.

The SR fans from the particles within $3 \sigma_{x}$ can not hit the beryllium pipe directly. The particles within $10 \sigma_{x}$ will contribute about $0.32 \mathrm{~W}$ of the SR power to the beryllium pipe. The background level for the IR design is lower than the limits set by the BESIII detector performance.

\section{IR SUPPORTING SYSTEM}

The SC magnet cryostat, transfer line, service cryostat and ISPB, Q1a, Q1b etc. are supported by a movable stage $^{[7]}$ shown in Fig.9. This movable stage could move along the beam line by approximately $3 \mathrm{~m}$. With this motion the SC magnets could pulled out and push into the detector.

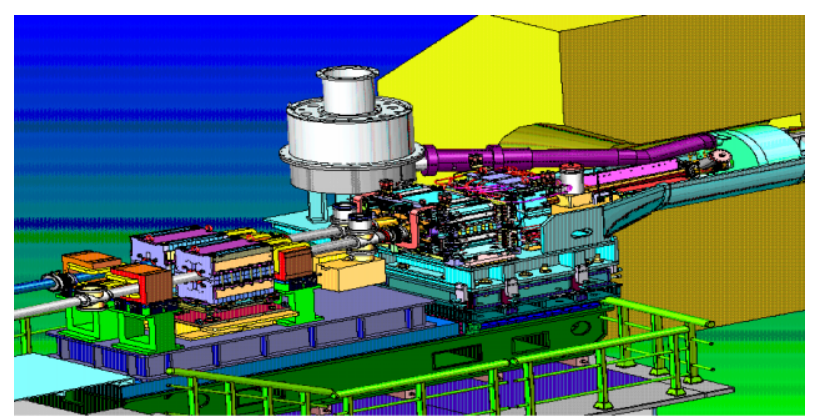

Figure 9: The supporting system of the IR magnets

The design of supporting system has been finalized with detailed drawings and structural analysis and its fabrication is underway.

\section{SUMMARY}

The IR design of BEPCII has been finalized. The special magnets ISPB and Q1a, Q1b have been successfully fabricated and field measurements show that their field performance meet the requirements of beam dynamics. The SC magnets and its valve box will be shipped to IHEP in August this year. We have started to think about the system assembly and commissioning issues of the SC magnets. Fabrication techniques for the support and vacuum chamber systems have been studied in some detail, and their fabrications are underway.

\section{REFERENCES}

[1] C. H. Yu, "Beam physics issues of the BEPCII interaction region", Proceedings of Mini-Workshop on the BEPCII Interaction Region., January 2004.

[2] J. B. Pang, "The design of septum bending magnet in the IR", Proceedings of Mini-Workshop on the BEPCII Interaction Region., January 2004.

[3] Brett Parker, "Review of operating currents, forces and torques for the BEPCII IR magnets", The 2'nd BEPCII Videoconference, May 2003.

[4] Z. S. Yin, "Design of the BEPCII IR septum quadrupole", Proceedings of Mini-Workshop on the BEPCII Interaction Region., January 2004.

[5] Y. Yang, "The design for the IR vacuum chamber", Proceedings of Mini-Workshop on the BEPCII Interaction Region., January 2004.

[6] X. W. Dai, "The heating analysis for the vacuum chambers in the IR", Proceedings of Mini-Workshop on the BEPCII Interaction Region., January 2004.

[7] M. T. Wang, "Support and vibration in the IR", Proceedings of Mini-Workshop on the BEPCII Interaction Region., January 2004. 